\title{
Adamantane derivatives having heterocyclic and monoterpenoid \\ residues as potent Tdp1 inhibitors
}

Aldar Munkuev (1), Alexandra Zakharenko (2), Evgeniy Suslov (1), Arina Chepanova (2), Konstantin Volcho (1,3), Nariman

\author{
Salakhutdinov $(1,3)$, Olga Lavrik $(2,3)$
}

1) N.N. Vorozhtsov Novosibirsk Institute of Organic Chemistry, Siberian Branch of Russian Academy of Sciences, 9, Lavrentiev Ave., Novosibirsk 630090, Russia

2) Institute of Chemical Biology and Fundamental Medicine, Siberian Branch of Russian Academy of Sciences, 8, Lavrentiev Ave., Novosibirsk 630090, Russia

3) Novosibirsk State University, 2, Pirogova Str., Novosibirsk 630090, Russia

Camptothecin derivatives, such as topotecan and irinotecan, are among the most common drugs being used in the treatment of cancer [1]. The mechanism of action of these drugs is associated with inhibiting topoisomerase 1 (Top1), an enzyme that plays an important role in cell division processes. However, there is a number of problems related to this type of therapy, in particular an ability of DNA repair system to remove damage caused by antitumor drugs. Tyrosyl-DNA phosphodiesterase 1 (Tdp1) is considered to play a key role in the repair of DNA lesions, thus preventing cancer cell death. Therefore, developing Tdp1 inhibitors is of great interest in modern medicinal chemistry as they could act synergistically with Top1 inhibitors in cancer combination therapy.

Adamantane derivatives as well as substances containing 1,3,4-thiadiazole and 1,2,4-triazole fragments have found wide application in medicinal chemistry $[2,3]$. On the other hand, structural modification of natural metabolites is one of the most fruitful approaches for the development of potential drugs. Monoterpenoids and their derivatives exhibit a number of biological activities, such as antibacterial, anti-inflammatory, antiviral, anticancer etc.

In an attempt to combine these structural blocks, namely adamantane, 1,2,4triazole/1,3,4-thiadiazole and monoterpenoid moieties in one molecule, we have synthesized compound I that has been transformed into the corresponding 1,3,4 thiadiazole II and 1,2,4-triazole III derivatives under acidic and alkaline conditions respectively, followed by the modification of heterocyclic compounds with monoterpenoid residues having acyclic, monocyclic or bicyclic structures.
Synthesis of carbocyclic acid derivatives

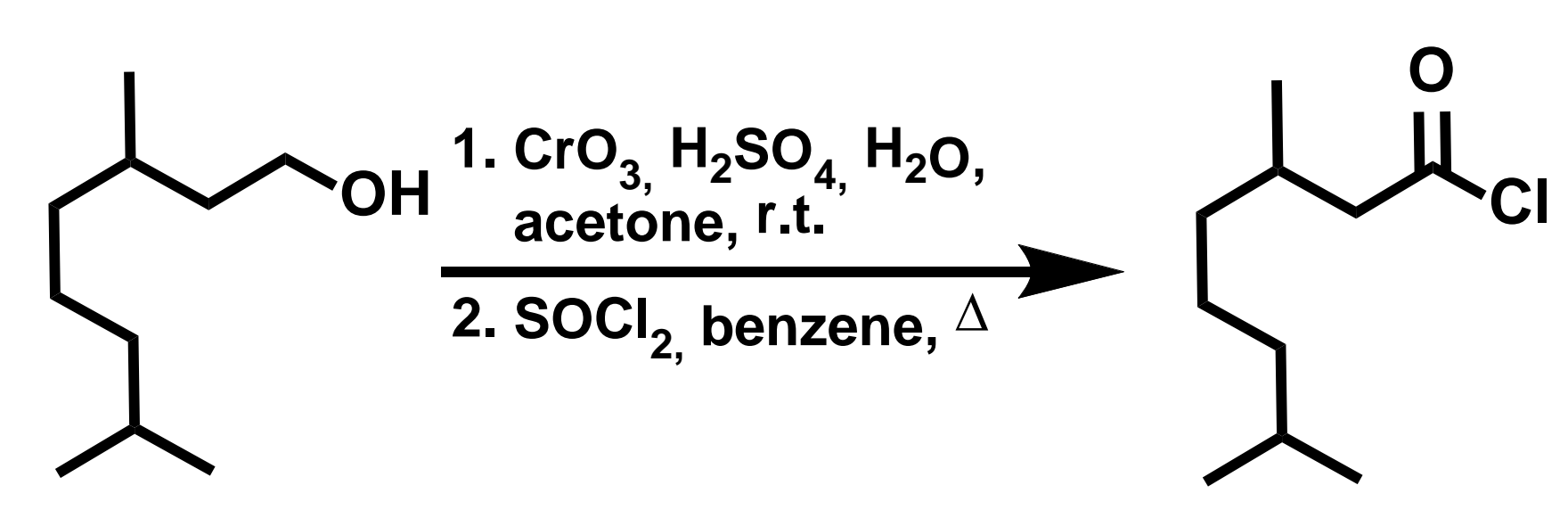
3,7-dimethyloctanoic acid 5 chloride $55 \%$<smiles>C=C(C)[C@@H]1CC=C(C=O)CC1CN[N+](=O)[O-]</smiles>

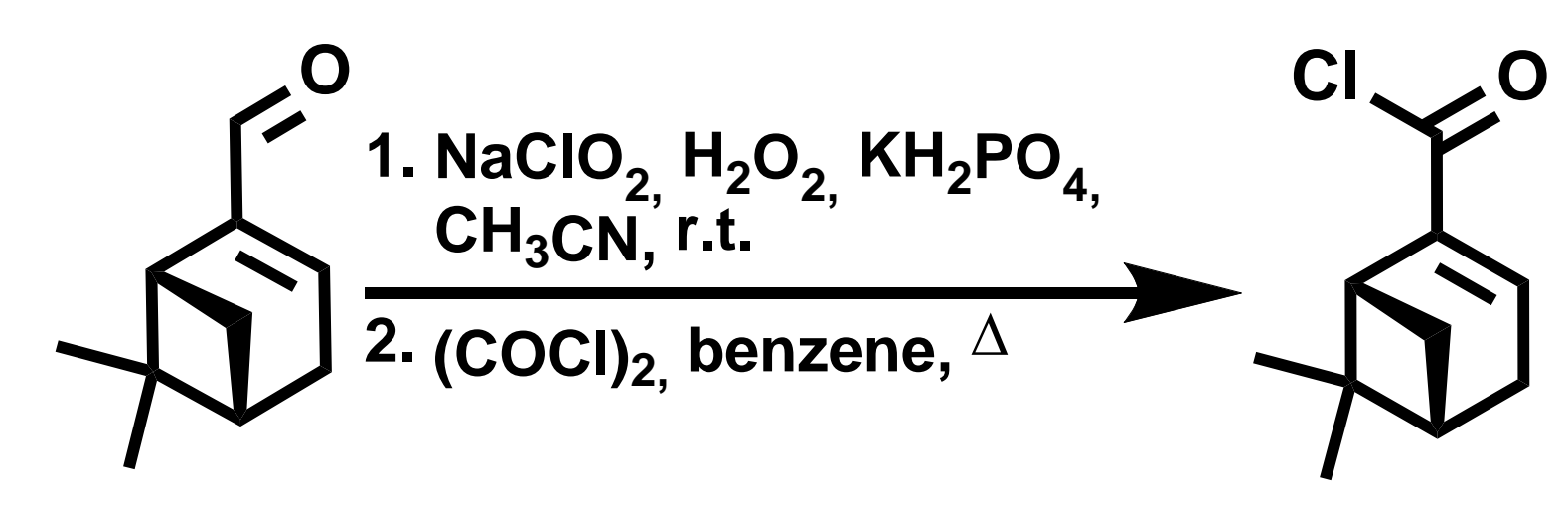
(-)-myrtenic acid chloride

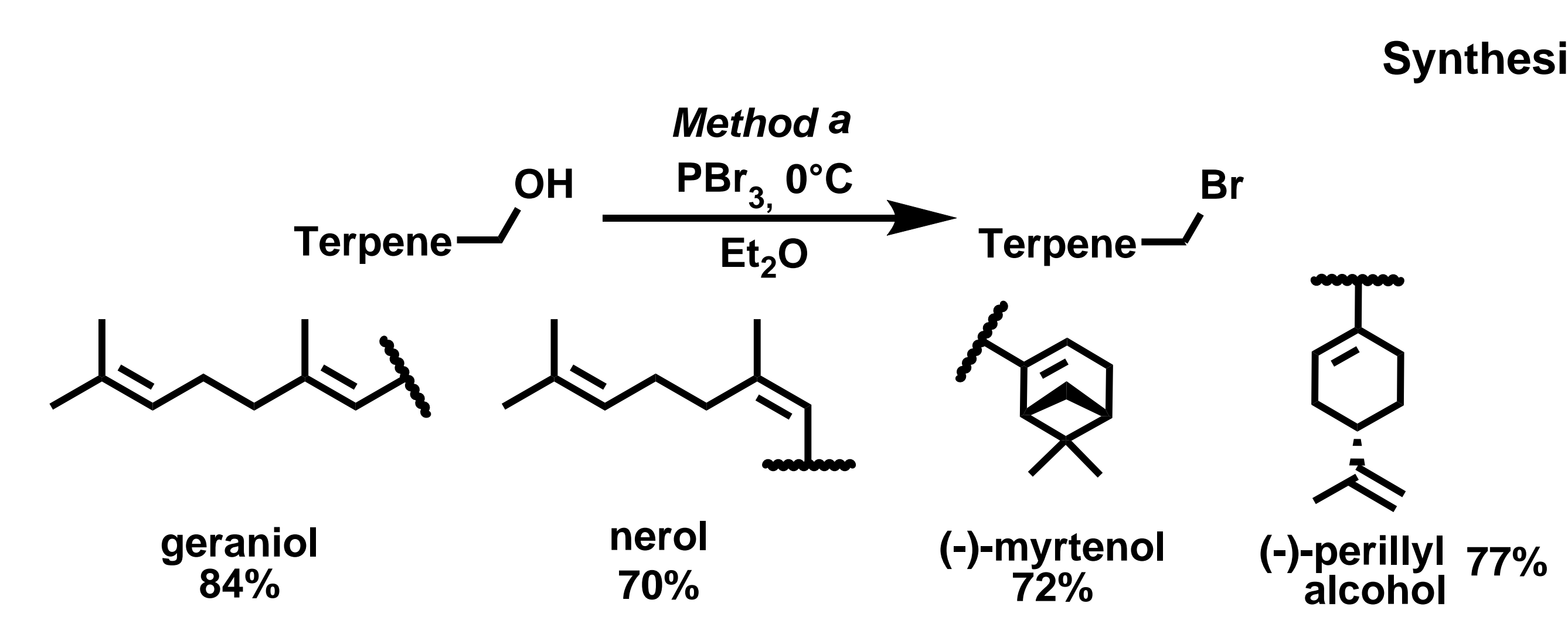

Synthesis of target compounds

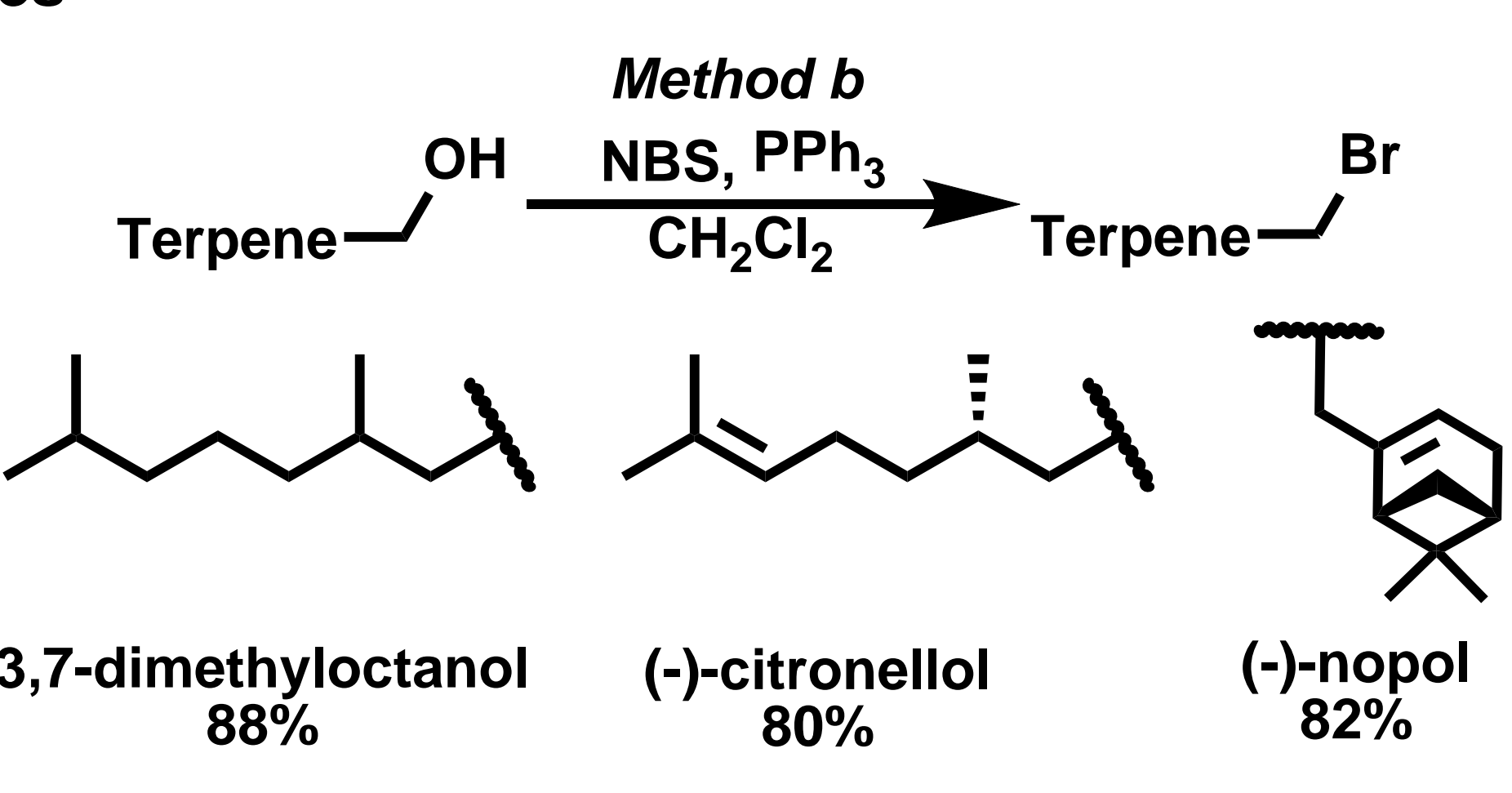

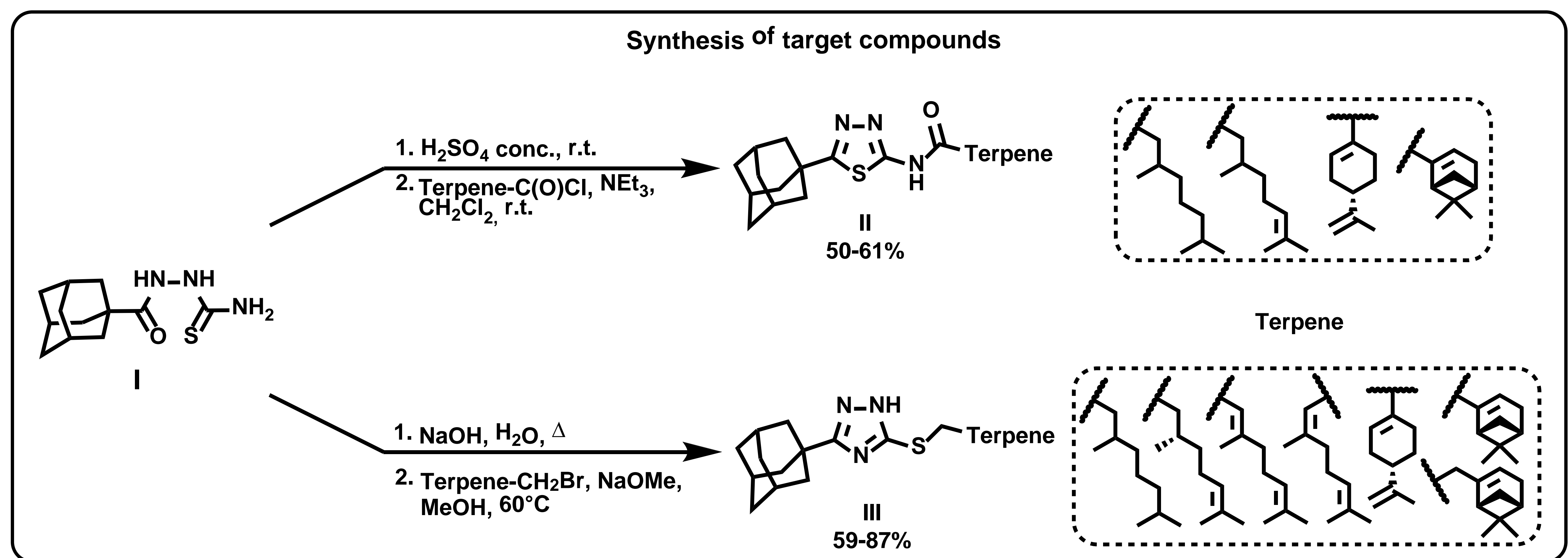

The compounds obtained were tested for their Tdp1 inhibitory properties. As depicted in Table 1, all the compounds were found to exhibit inhibitory activity at submicromolar and micromolar concentrations.

\begin{tabular}{|c|c|c|c|}
\hline Compound & $\mathrm{IC}_{50, \mathrm{HM}}$ & Compound & $\mathrm{IC}_{50,}, \mathrm{\mu M}$ \\
\hline 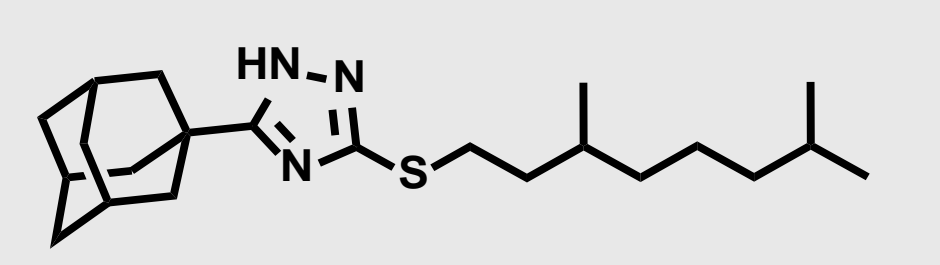 & $0.54 \pm 0.09$ & 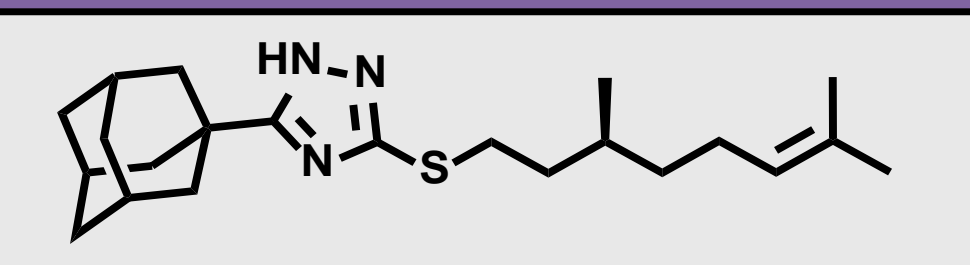 & $1.50 \pm 0.30$ \\
\hline 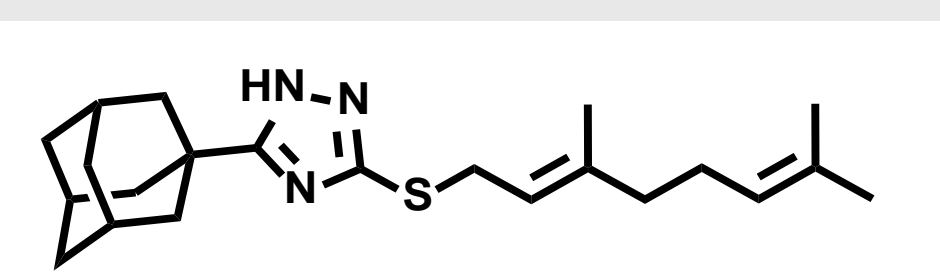 & $5.30 \pm 1.70$ & 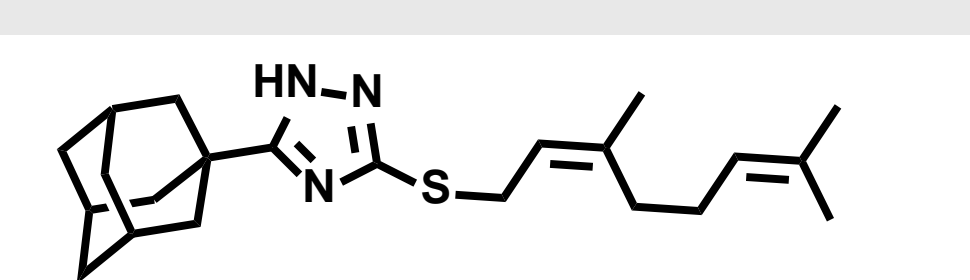 & $5.60 \pm 0.60$ \\
\hline $\mathbb{8}$ & $6.20 \pm 2.20$ & 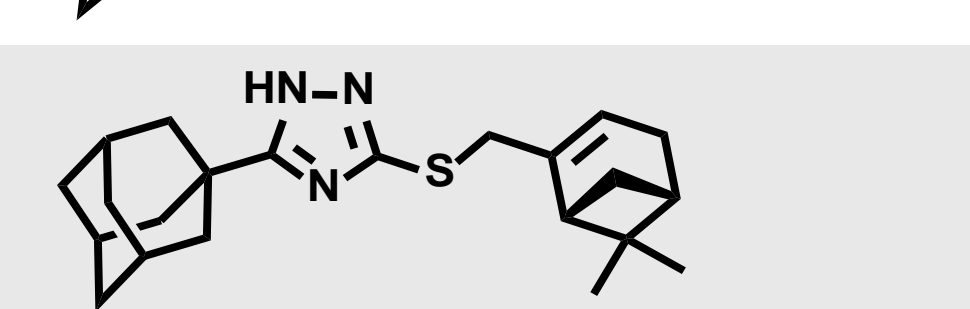 & $7.50 \pm 1.80$ \\
\hline 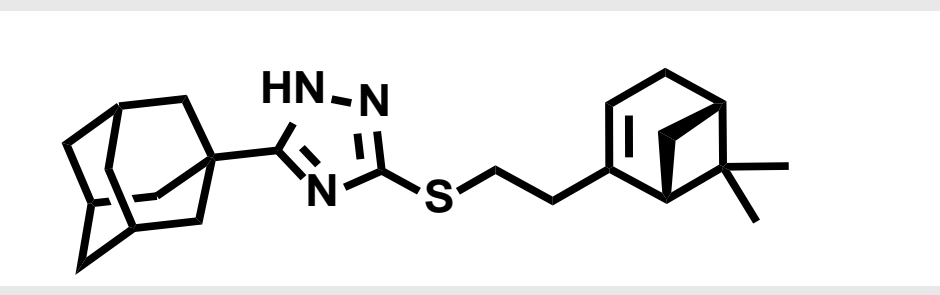 & $0.57 \pm 0.14$ & 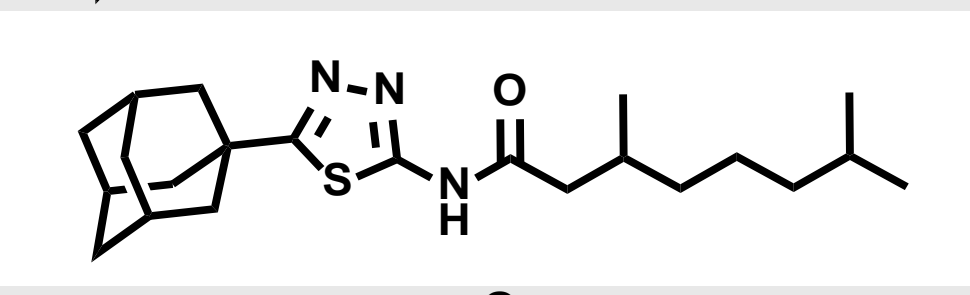 & $0.35 \pm 0.05$ \\
\hline E- & $2.59 \pm 0.48$ & 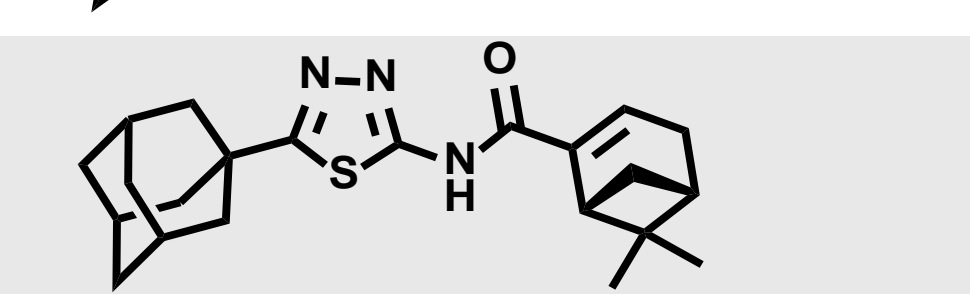 & $0.45 \pm 0.09$ \\
\hline
\end{tabular}

Among thiadiazoles II, the amides of 3,7-dimethyloctanoic or (-)-myrtenic acids demonstrated the most pronounced activity, with $\mathrm{IC}_{50}$ being $0.35 \pm 0.05$ and $0.45 \pm 0.09 \mu \mathrm{M}$, respectively. As for triazoles III, the highest $\mathrm{IC}_{50}$ value was found for compounds containing (-)-nopol $(0.57 \pm 0.14 \mu \mathrm{M})$ and 3,7-dimethyloctanol $(0.54 \pm 0.09 \mu \mathrm{M})$ moieties. It should be noted that (-)-myrtenyl derivative of 1,2,4triazole III proved to be the least active with an inhibitory effect on Tdp1 at a concentration of $7.50 \pm 1.80 \mu \mathrm{M}$, whereas thiadiazole II compound having the analogous substituent showed the best inhibitory properties. In general, amide 1,3,4-thiadiazoles II demonstrated higher activity compared to 1,2,4-triazoles III. In conclusion, we have found the promising compounds capable of inhibiting Tdp1 at submicromolar/micromolar concentrations. The study can aid in the development of new compounds that can potentiate the cytotoxicity of Top1 inhibitors toward cancer cells. 